\title{
Decoding neural correlates of cognitive states to enhance driving experience
}

\author{
Ricardo Chavarriaga $^{\dagger}$, Marija Ušćumlić ${ }^{\dagger, \ddagger}$, Huaijian Zhang ${ }^{\dagger, \ddagger}$, Zahra Khaliliardali ${ }^{\dagger}$, \\ Ruslan Aydarkhanov $^{\dagger}$, Sareh Saeedi ${ }^{\dagger,}$, Lucian Gheorghe ${ }^{\dagger, \ddagger}$, José del R. Millán ${ }^{\dagger}$ \\ † Chair in Brain-Machine Interface, École Polytechnique Fédérale de Lausanne, EPFL, Geneva, Switzerland \\ ¥ Nissan Motor, Mobility Services Laboratory, Nissan Research Center, Atsugi, Japan \\ ricardo.chavarriaga@epfl.ch
}

\begin{abstract}
Modern cars can support their drivers by assessing and performing autonomously different driving maneuvers, based on information gathered by in-car sensors. We propose that brain machine interfaces (BMIs) can provide complementary information that can ease the interaction with intelligent cars in order to enhance the driving experience. In our approach, the human remains in control, while a BMI is used to monitor the driver's cognitive state and use that information to modulate the assistance provided by the intelligent car. In this review, we gather our proof-of-concept studies demonstrating the feasibility of decoding electroencephalography (EEG) correlates of upcoming actions and those reflecting whether the decisions of driving assistant systems are in-line with the driver intentions. Experimental results while driving both simulated and real cars consistently showed neural signatures of anticipation, movement preparation and error processing. Remarkably, despite the increased noise inherent to real scenarios, these signals can be decoded on a single-trial basis, reflecting some of the cognitive processes that take place while driving. However, moderate decoding performance compared to the controlled experimental BMI paradigms indicate there exists room for improvement of the machine learning methods typically used in the state-ofthe-art BMIs. We foresee that fusion of neural correlates with information extracted from other physiological measures; e.g. eye movements or electromyography (EMG) as well as contextual information gathered by in-car sensors will allow intelligent cars to provide timely and tailored assistance only if it is required; thus keeping the user in the loop and allowing him to fully enjoy the driving experience.
\end{abstract}

Keywords-Brain-machine interface, EEG, Error-related potentials, Anticipation, Movement related potentials, slow cortical potentials, Classification, Car simulator

\section{INTRODUCTION}

In recent years, there has been an impressive development in technology-enabled intelligent cars. By processing the environment, these cars can provide their drivers with extra information (e.g. distance to the nearest car) or autonomously assess and perform different driving maneuvers, including lane changing assistance, parking or even fully autonomous navigation. Although these features improve the driving experience, we believe that an optimal car-driver symbiotic interaction would be fully achieved once the car can also take into account the driver's goals and preferences. In the case of a lane change, if a car can recognize a driver's intention to change the lane, the car's assistance system can better align its behavior to the driver's one and provide them with a suitable and timely assistance when executing the action.

To communicate driver's cognitive processes to cars, we propose the use of non-invasive brain-machine interfaces (BMIs) as illustrated in Figure 1. In this review, as a proof of concept, we bring together the studies on the transfer of the state-of-the-art BMI to the driving application. These works demonstrated the feasibility of electroencephalography (EEG) -based decoding of cognitive processes in simulated and real-car driving scenarios. It is worth noticing that recording and decoding EEG signals during driving poses a particular challenge compared to the conventional BMI experiments. The latter are typically performed in well-controlled laboratory conditions: based on simple tasks (e.g. finger movement, anticipation of simple objects in a sequence) and minimizing potential sources of signal contamination such as body and eye movements, and environmental noise [1], [2]. In consequence, development of BMI systems suited for driving scenarios represents a unique opportunity for improving the signal processing and decoding methods typically used in the field.

The focus of this review is on decoding the cognitive processes of movement preparation, anticipation and error monitoring in driving. Movement preparation is a cognitive process preceding the execution of any motor actions, such as steering or pressing a pedal in driving. Decoding of the corresponding neural correlates would allow a car to infer ahead of time about the drivers' intention to act. Moreover, the neural correlates of anticipation can be informative about driver's future behavior in dynamic driving environments, where their actions are led by external events, such as traffic light changes - a driver anticipates the appearance of the color requiring certain action from him, namely the actions of brake/accelerate which have to be executed upon the transition green-yellow-red or vice versa. Complementarily, decoding the neural correlates of error monitoring is particularly interesting for closing the loop in a driver-car symbiotic interaction. This cognitive process can reflect whether the car behavior coincided with the driver's intention, what may have strong impact on adapting the assistance the intelligent vehicle can provide (e.g. the maneuvers it can perform autonomously).

Before entering in the driving-related BMI studies (Sections III-V), we make a concise and general overview of the BMI framework for driving in Section II. First, we review existing 
work on decoding neural correlates of drowsiness, workload and emergency actions (Section II-A), and then we discuss cognitive processes that can be used for enhancing humanmachine symbiosis (Section II-B).

\section{BRAIN-MACHINE INTERFACES FOR DRIVING}

\section{A. Drowsiness, workload and emergency}

Previous studies on BMI systems for drivers have focused on brain correlates of the level of drowsiness and workload [3]-[10]. Driving, although a part of our daily routine, requires a high level of alertness and responsiveness that often can be affected by secondary activities (e.g. communication with a fellow passenger, using a navigation system) or different mental states (e.g. drowsiness after a long ride). Consequently, the idea of monitoring the driver's state using EEG and other physiological signals has been proposed as a convenient tool for obtaining an objective assessment of workload, vigilance or drowsiness [11]. Some of the challenges of this research line are the definition of the states to be detected (e.g. awake, drowsy, and sleep), the ability to modulate in a controlled way the mental states by the experimental conditions (often made through changes in the driving conditions or by introducing secondary tasks [3], [12]), and obtaining reliable ground truth labels. The latter process usually relies on the subjective post-experiment self-assessment made by participants using questionnaires (e.g. NASA-TLX [13]) which limits the reliability and temporal resolution at which such labels can be obtained [14].

A large body of studies have pointed out to changes in alpha and theta waves related to fluctuations in vigilance and performance degradation. This is complemented by increases in beta oscillations associated with increased alertness and arousal, as well as increase alpha activity for decreased attention levels. A review of these correlates can be found in reference [11]. Nonetheless, there is not yet a full characterization of how consistent these modulations are across different tasks or subjects. Hence, the generalization of these results over a wide range of scenarios is still to be assessed. Based on these studies, spectral EEG analysis is typically used for studying the neural correlates of drowsiness and vigilance [8]. Khushaba and colleagues extracted features using a Fuzzy mutual-information based wavelet packet transform on a combination of EEG, electrooculography (EOG) and electrocardiogram (ECG) signals recorded during simulated driving [4]. Alternatively, other researchers have explored whether different levels of fatigue could be discriminated based on interactions among different brain areas estimated using Granger causality [7], transfer entropy [6], or partial directed coherence [15]. Another approach has looked at the stability of the ICA-estimated neural sources [16].

A wide range of classification methods has been applied for EEG-based mental state detection - from simple classifier models such as Linear Discriminant Analysis (LDA) [4], [17], and Support Vector Machines [18], [19] to artificial neural networks [10], [19]-[21]. Interestingly, some research groups have evaluated the possibility of applying transfer learning methods to reduce the amount of subject-specific data required to calibrate the EEG decoder [8], [9]. Recent studies performing simultaneous EEG and functional nearinfrared spectroscopy (fNIRS) recordings showed promising results on the use of the latter modality to obtain information about driver's drowsiness [17], [22]. Upon recognition of these mental states, the driving assistance system can make actions aimed at improving driver alertness [23]. One proposal is to use personalized music recommendation as the feedback when a higher level of drowsiness is detected [19]. Nonetheless, the efficacy of these approaches is yet to be evaluated.

Another driving situation that has been studied for potential use of BMI systems is emergency situations. In these cases, there is an external event (e.g. sudden braking of the front car) initiating a driver's reaction. Recognition of such critical event followed by an appropriate action selection and execution is reflected in the evoked potentials in the driver's EEG as shown in the study [24]. Moreover, the authors demonstrated that this EEG signature can be detected about $130 \mathrm{~ms}$ before the actual braking, discussing its usage in prevention of traffic accidents by integrating it in braking assistance systems. The authors verified the results in a real-car driving task [25], and further explored real-world driving challenges by considering diverse braking situations in simulated driving [26]. In the latter study, the authors proposed a combination of EEG features - the readiness potential and event-related desynchronization together with the ERPs - for improved decoding performance using the regularized linear discriminant analysis.

\section{B. Decoding cognitive processes for driver-car symbiosis}

Complementing the above described BMI approaches for driving, we address the possibility of decoding brain correlates of cognitive states reflecting upcoming actions and driver's perception of the feedback provided by the car. We aim to use these signals to predict future actions and/or evaluate whether the decisions of the intelligent system are coherent with the user intentions.

BMI-supported driving assistance requires the interpretation of the neural signals in real-time. To realize efficient decoding of the driver's cognitive processes from high-dimensional neural data, despite the high trial-to-trial variability, it is necessary to exploit tools and methods from both neuroscience and engineering. In particular, to identify appropriate features (i.e. brain areas and patterns of activity that are actually related to the phenomenon of interest), we combine data-driven approaches and existing knowledge about brain organization. In the reported studies, we used commonly applied decoding techniques in state-of-the-art closed-loop BMI studies. Although more advanced decoding techniques are promising, their application is often limited due to the small number of training samples available in BMI scenarios. Nonetheless, simple techniques have low computational complexity that is critical for real-time applications while preserving a satisfactory single-trial decoding performance [27], [28].

Concerning cognitive processes of interest in the frame of BMI-mediated car-driver symbiosis, it has been largely reported that anticipatory and preparatory processes are reflected in slow negative potentials, preceding the anticipated 


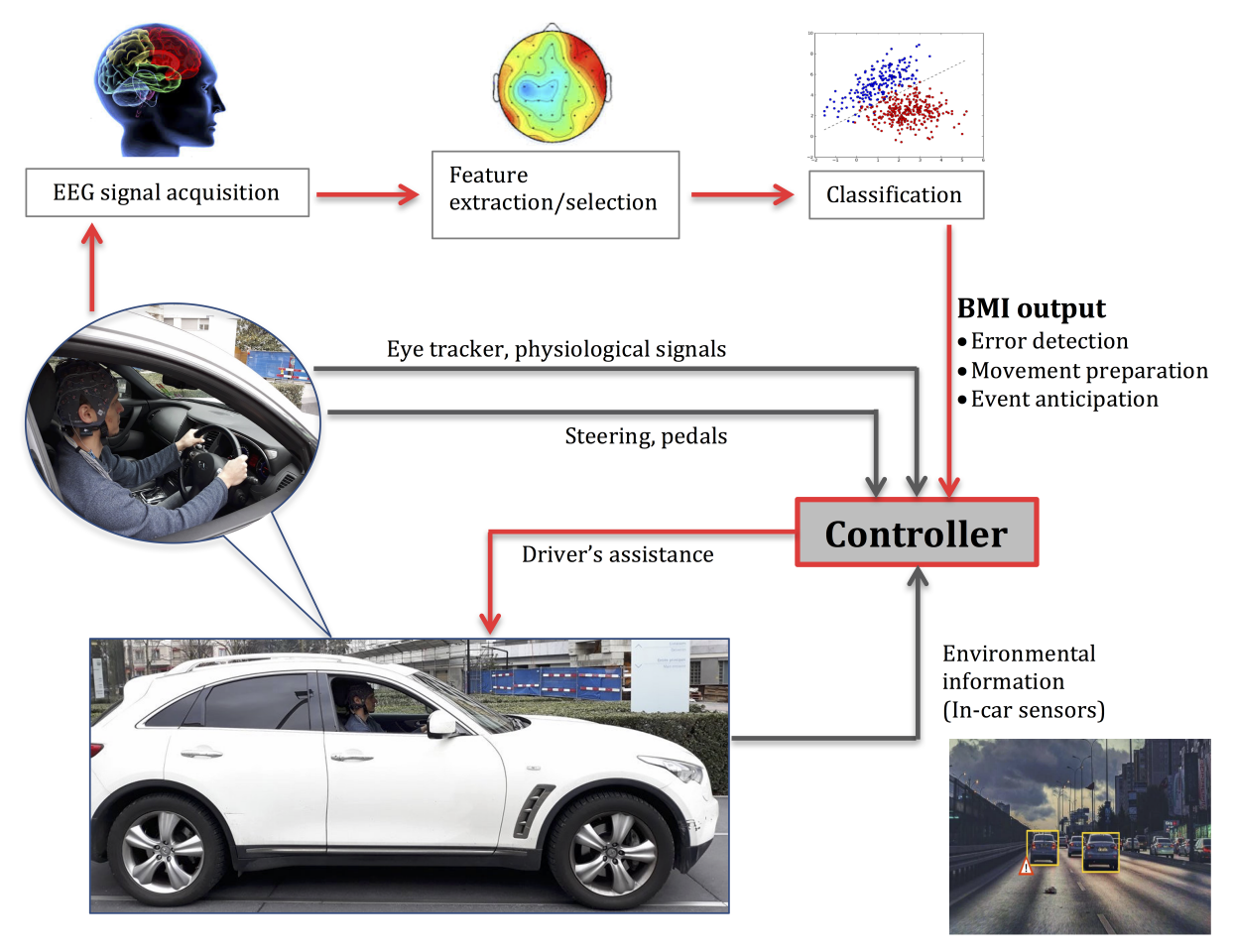

Fig. 1. Overview of the BMI-supported car-driving symbiotic approach. The controller of the intelligent car takes into account environmental information, driver's physiological signals, as well as their cognitive states inferred by the BMI from EEG to decide on the type and level of assistance it provides. This review shows examples of potential information that can be extracted by the BMI system for closing the driver-vehicle loop (denoted by red arrows).

stimuli or the motor action. The anticipation-related potential is commonly referred to as the Contingent Negative Variation $(\mathrm{CNV})[29]$ and has been shown as a slow negative EEG deflection in fronto-central areas that starts developing after a warning cue. A vast amount of studies report the presence of these processes using simple experimental protocols and, more recently, evidence about their presence in realistic applications such as web-browsing application protocol and driving is emerging [30]-[32]. Regarding EEG signals related to preparatory processes, the so-called motor related cortical potential (MRCP) $)^{1}$ corresponds to a negative deflection of the EEG activity over motor areas appearing as early as 1s before the movement onset [33]. Single-trial analysis of these signals can be used to extract information about upcoming actions for both upper and lower limbs [34], [35]. Evidence that such correlates can be successfully decoded in a singletrial basis [30] shows that they can provide markers of the user's preparedness to execute a given action.

The existence of anticipation- and preparatory- potentials elicited while driving both in a car simulator and a real vehicle allows to infer upcoming driving actions. This can complement information from other sources (e.g. in-car sensors or other physiological signals) to decide the type of assistance required by the user in that context [36]. Here, we present our work in this line proving the feasibility of detection of the cognitionrelated signatures in the EEG while driving, what was previously demonstrated in some other realistic applications such

\footnotetext{
${ }^{1}$ Also referred to as readiness potential or Bereitschaft potential.
}

as a web-browsing application, a navigation task and a selfpaced reaching movement [30], [32], [35], [37]. Moreover, the presented work contributes to the perspective on the blending of human and machine intelligences through shared control [38].

These decisions, however, may be prone to errors which may hinder performance. Previous works in BMI have proposed the decoding of human perception of machine decisions as a means to improve performance [39]. In particular, errorrelated brain activity provides information on whether the user agrees with the actions of an external device and can be exploited to adapt such device to tailor the user's goals and preferences [39], [40]. We studied this approach in a scenario where a driving assistance system provides feedback on predicted upcoming turning directions (e.g. based on environmental information) [41].

This paper reviews recent results showing that both actionand error-related brain responses observed during driving are consistent with previous studies in simpler experimental protocols. Furthermore, these signals can be decoded in a single-trial basis above chance levels, thus making it possible to infer the driver's cognitive processes. Overall, these results validate the consistency of the reported brain signals, and provide initial evidence supporting the possibility of using them in practical applications. 


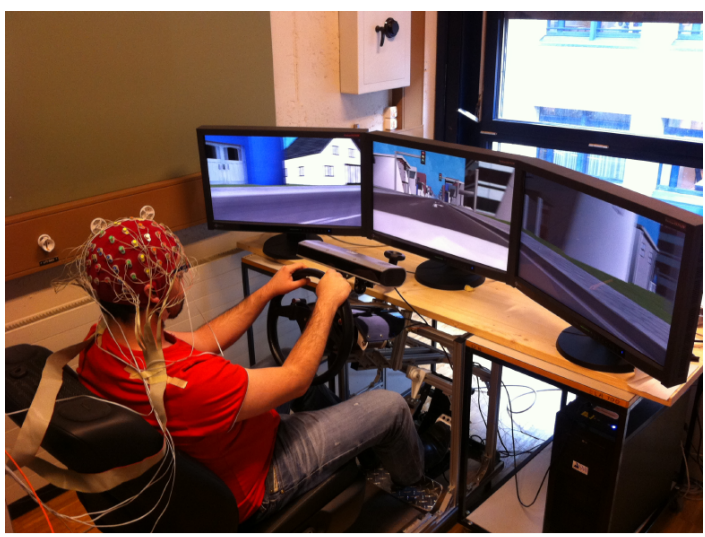

Fig. 2. Driving simulator used in the experiments. The subject drives a simulated vehicle through a simulated environment shown in three $3 \mathrm{D}$ screens while wearing a 64-channel EEG acquisition system.

\section{EXPERIMENTAL SET-UP}

Experiments were performed in both a realistic car simulator and a real car. The custom-made simulated car, shown in Figure 2, corresponds to a small size vehicle. A passive system based on springs and fluid damping was used to generate a realistic steering feeling (electrical force feedback were avoided to reduce potential contamination of EEG recordings). A virtual driving scene is presented to the subject using three 27" 3D monitors. These monitors do not require the subject to wear glasses and present the scene from 8 points of view, reproducing not only binocular parallax, but also motion parallax (i.e. the scene changes accordingly if subjects move their head sideways). The simulator continuously records the steering and pedal positions, vehicle dynamics and the position of the vehicle in space.

Experiments in the real car setup were performed in an Infiniti FX30 vehicle, Figure 1. This car has an automatic gearshift, therefore subject's arm movements were limited to steering actions. Although the car provides several driving assistance systems -intelligent cruise control, lane departure prevention and vehicle dynamic control- they were disabled during the experiments. The vehicle has been fitted with a driving logger (provided by the manufacturer) that records steering, pedal positions and vehicle dynamics. This information is obtained directly from the in-car CAN bus. For safety reasons, real driving experiments were performed in a closed road with no other vehicles. Traffic lights and signs were specifically deployed according to the experimental protocols (see below).

EEG signals of the driver were recorded at $2048 \mathrm{~Hz}$ using a 64 channel BioSemi ActiveTwo System, in an extended $10 / 20$ layout configuration. Signals were then downsampled to $256 \mathrm{~Hz}$. Eye movement activity was simultaneously recorded using electrodes positioned above the nasion and below the outer canthi of both eyes. Furthermore, one bipolar electromyography (EMG) channel was placed on the driver's right leg (tibialis anterior muscle) to monitor pedal activity. EMG signals were band-pass filtered in the range [20 50] $\mathrm{Hz}$ and smoothed using a moving average ( $\mathrm{t}=25$ samples).

In both the real and simulated setups, recordings were

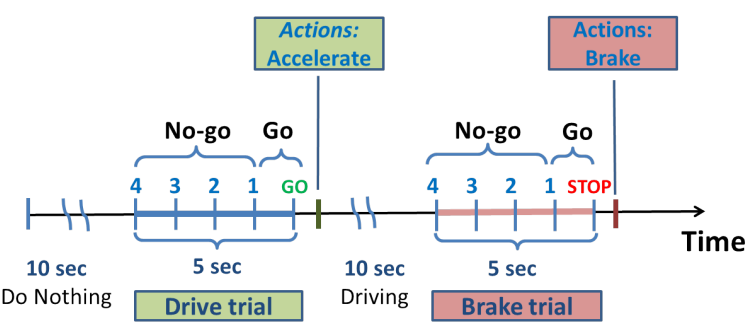

(a)
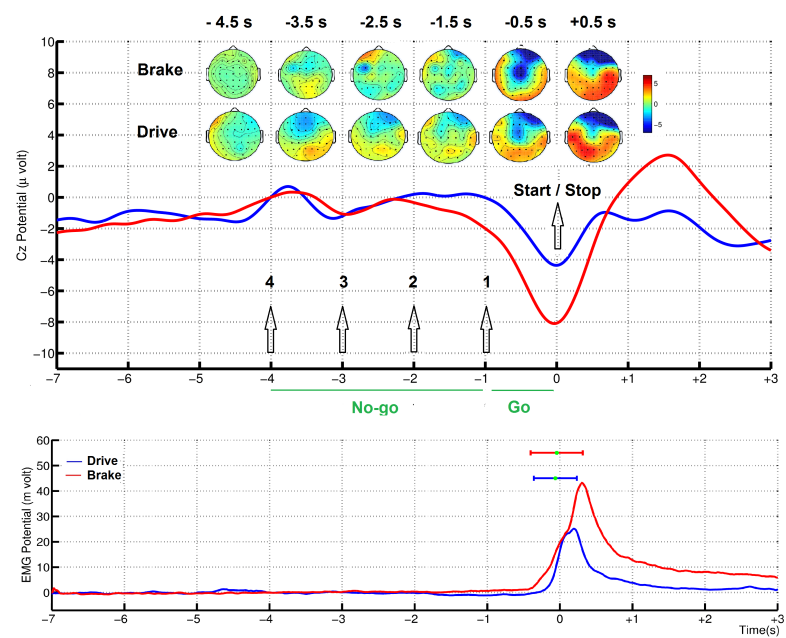

(b)

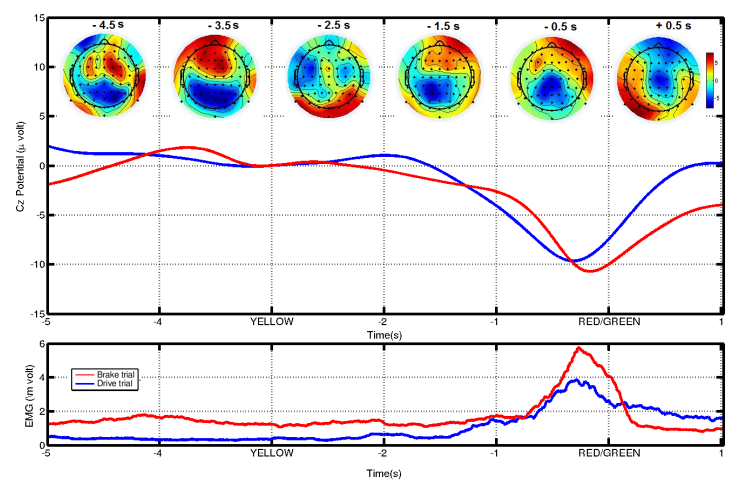

(c)

Fig. 3. Anticipation-related potentials. (a) Experimental protocol in the simulated driving. (b-c) Grand average ERP (Cz electrode) of an exemplar subject for both brake and accelerate conditions (red and blue lines, respectively). The negative EEG deflection can be clearly observed after the last warning stimulus (Go condition; [-1 0] s period), but not in the preceding ones (No-go condition; [-3 -1] s period). (b) Simulated driving. Bottom plot shows EMG activity. (c) Real car driving.

synchronized via hardware using a pulse train (frequency $256 \mathrm{~Hz}$ ) sent from the driving simulator or car logger to the EEG recording device via parallel port.

\section{BRAIN CORRELATES OF UPCOMING DRIVING ACTIONS}

\section{A. Actions driven by environmental cues}

During driving, environmental cues warn the driver about upcoming situations where an action may be required (e.g. accelerate when a traffic light changes to green). The existence 
of anticipation-related signals in such driving situations was demonstrated both in simulated and real driving.

In the simulated experiment, subjects $(\mathrm{N}=18)$ were driving along a highway at $100 \mathrm{Km} / \mathrm{h}$ and their task was to accelerate or brake at specific points cued by visual stimuli ('GO' or 'STOP') [31]. The visual stimuli consist of a numerical countdown starting at '4' shown in the middle of the screen, Figure 3(a). The countdown finishes with the cue to the required action to be performed. This setting enables us to evaluate brain responses in two conditions depending on the required upcoming behavior. On the one hand, there are periods where no action (i.e. braking or accelerating) is required after the next stimulus (i.e. No-Go condition: in the period between the stimuli '4' and ' 1 '). On the other hand, in the remaining condition (between the '1' stimulus and the GO cue) the subject should prepare to quickly act at the appearance of the final stimulus (Go condition). This experiment yielded about 360 instances of accelerating and braking situations per subject, with their corresponding 'GO' and 'No-Go' trials. It is worth to notice that the appearance of the warning stimuli allows the driver to anticipate and prepare for the upcoming action, opposite to the emergency brake behavior tested by Haufe and colleagues [24], [25].

In the real car experiment, subjects $(\mathrm{N}=8)$ were asked to drive at maximum $65 \mathrm{Km} / \mathrm{h}$ and safely brake or accelerate as soon as the traffic light changed color to red or green respectively [42]. In this case the period when the yellow light is on before turning to red or green corresponds to the 'Go' condition for the brake or accelerate trials. In both cases, the yellow light stayed on for three seconds. The traffic lights were deployed to ensure that they will turn to red before the car reached their location without requiring sudden brakes.

Based on previous studies, we focused on the slow cortical potentials (SCP, [0.1 1] Hz). Both in the simulated and real driving experiments we found EEG correlates of anticipation and movement preparation that are consistent with signals previously reported in much simpler setups. Namely, a centromedial slow negative signal can be observed well before anticipated actions (accelerate/brake) both in simulated and real driving, c.f., Figures 3(b) and 3(c).

In order to test single-trial detection of EEG activity reflecting the action anticipation, we created two types of trials corresponding to those periods before an action is performed (i.e. Go trials), and those when no response is required (i.e. No-Go trials). We then use a Quadratic Discriminant Analysis (QDA) classifier to discriminate these two types of trials [31]. The feature vector consisted of the EEG activity at the location of the $\mathrm{Cz}$ electrode at time-points $800 \mathrm{~ms}, 600 \mathrm{~ms}, 400 \mathrm{~ms}$ and $200 \mathrm{~ms}$ before the end of the trial. EEG data was spatially filtered using a Common Average Reference (CAR) and a smoothing Weighted Average Filter (WAVG) [30], prior to the spectral filtering between $0.1 \mathrm{~Hz}$ and $1 \mathrm{~Hz}$.

The performance evaluation of the learned models was done using a sliding window starting from 6 seconds before the onset of the imperative stimulus. The results are reported with reference to the chance level obtained at each time point by shuffling the labels of the training data and performing 1000 times 4-fold cross validation.
In the driving simulator, the average classification performance in terms of the Area Under the specificity-sensitivity Curve (AUC) using temporal features up to $200 \mathrm{~ms}$ before the action was $0.78 \pm 0.04$ and $0.83 \pm 0.05$ for accelerating and braking actions, respectively ( $\mathrm{N}=18$; 4-fold cross-validation). The choice of a classification window finishing $200 \mathrm{~ms}$ before the possible action is motivated by the need to decode potential actions before they happen to provide enough time for a potential driving assistance system to perform the classification and take a corresponding action if required. Preliminary results in the in-car experiment suggest that these signals can also be discriminated in the real scenario. For the subject shown in Figure 3(c) the AUC values are 0.88 and 0.72 for the acceleration and brake trials, respectively.

\section{B. Self-generated actions}

We also studied neural activity preceding self-generated actions and assessed the possibility of decoding it to detect whether the driver would perform a lane change in the immediate future. We analyzed the EEG activity while drivers $(\mathrm{N}=6)$ performed self-paced lane changes (LC) in a simulated highway [43]. In this experiment, subjects drive in an ovalshaped road and change the driving lane during the straight segments at the moment of their choice. No other cars were included in the simulation.

The EEG data was filtered between $0.1 \mathrm{~Hz}$ and $1 \mathrm{~Hz}$ to analyze SCPs and segmented into trials corresponding to straight driving and steering actions based on the driving simulator data; c.f., Figure 4(a). We used as features the EEG activity at the positions of six channels $(\mathrm{C} 1, \mathrm{Cz}, \mathrm{C} 2, \mathrm{CP} 1, \mathrm{CPz}, \mathrm{CP} 2)$ within a time window preceding the LCs and LDA was used for classification. As seen in Figure 4(b), slow negative EEG deflections appear over central areas, which is consistent with the movement-related potentials before $500 \mathrm{~ms}$ prior to lane changes. Decoding performance yielded a true positive rate of $79.4 \pm 9.2$ (5-fold cross-validation), with average detection times of $613 \pm 169 \mathrm{~ms}$ before the actual steering action.

\section{Driver's response variability}

A critical factor for any intelligent car is timely assistance that matches the driver's behavior and intention. To this end, we performed an EEG study on the neural basis of stimulus driven behavior during car driving. In particular, we addressed the neural markers of the response variability in an obstacle avoidance driving task [44]. We explored how EEG activity, elicited by the appearance of obstacles which required lane changes, relate to the steering reaction. These correlates could be used to track neural processes that influence changes in the driver's response time and allow the intelligent car to adapt to the driver's response variability in diverse road situations.

In this experiment, subjects $(\mathrm{N}=15)$ were asked to continuously drive at $95 \mathrm{Km} / \mathrm{h}$ along a virtual two-lane road avoiding the obstacles. Appearance of an obstacle on the road would require an action (i.e. lane changing) only if it was located in the same lane as the car. During the experiment, the probability of this situation was $25 \%$. The distance between the car and an obstacle at the moment of its appearance varied 

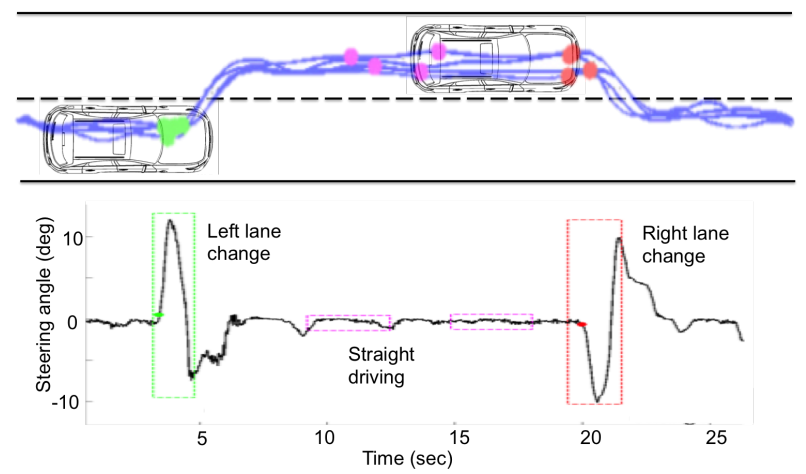

(a)
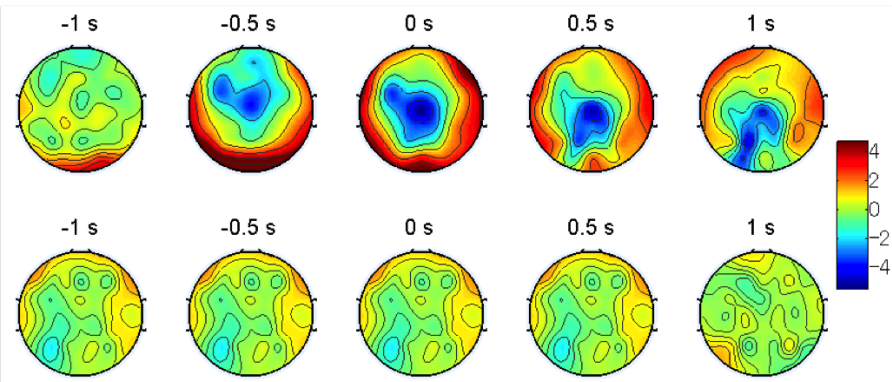

(b)

Fig. 4. Movement-related cortical activity preceding lane changes. (a) Car trajectories in one straight segment of the simulated highway are shown in the top plot. Blue traces correspond to single laps. Dots represent different conditions depending on the steering angle (bottom). Green: Change towards the left ; Red: lane change towards the right; Magenta: Straight driving. Bottom: Steering pattern. (b) Grand average ERP during lane changes (top) and straight driving periods (bottom). Topographical activity represented by a top view of the scalp (nose up). $\mathrm{t}=0$ corresponds to the moment of steering.

randomly between $40 \mathrm{~m}$ and $70 \mathrm{~m}$ (uniformly distributed) and the time between two consecutive obstacles was about $5 \mathrm{~s}$. The variability in the distance to the obstacle elicited different types of drivers' reactions. Obstacles at close distance provoked rapid responses, while those appearing in the far distance led to slower and self-paced responses.

The results showed EEG-based neural markers of steering response variability in both time- and spectral- domains. A significant correlation between the peak latency of the eventrelated potentials (the EEG data filtered between 1-10 Hz) and the onset of the steering behavior was found in frontal and parietal areas. Moreover, reaction time was significantly correlated with theta power modulation $(4-8 \mathrm{~Hz})$ in the frontocentral $(\rho=-0.3$ in FCz; $p<0.005)$ and parietal $(\rho=-0.34$ in $\mathrm{CPz} ; p<0.001)$ regions in a way that stronger increase in theta reflects faster reactions. The $p$ values were obtained using Student's t-distribution with the assumption of bivariate normal distribution. A positive correlation was found between the beta power modulation $(20-35 \mathrm{~Hz})$ and the reaction time ( $\rho=0.2$ and 0.18 for $\mathrm{FCz}$ and $\mathrm{CPz}$ respectively), suggesting that the faster the reaction, the more decrease in beta. However, these correlations were not statistically significant $(p>0.05)$.

\section{ERROR-RELATED BRAIN POTENTIALS IN HUMAN-CAR INTERACTION}

As mentioned earlier, error-related brain activity provides information on whether the user agrees with the actions of an external device and can be exploited to adapt such device to the user's goals [39], [40]. We evaluated how the same approach can be used in intelligent cars. In this case, we assume the car is endowed with a means to infer driver's behavior (e.g. directions of turning at intersections) and uses this information to assist in the upcoming maneuvers. The driving assistance system can inform the driver about its inferences and the elicited brain response is used to confirm or refute such inference [32].

The experiments were performed in both the car simulator $(\mathrm{N}=23)$ and the real car $(\mathrm{N}=8)$ [41]. In both cases, traffic signs showed the driver the designated path (i.e. the direction in which they should turn at the next intersection). When approaching an intersection, the car displays visual stimuli (i.e. arrows) that indicate its inference about the next direction of turning, as shown in Figure 5(a). Erroneous feedback pointing to a different direction than the one marked by the signs - was presented $30 \%$ of the times. The experiment yielded about 150 trials per subject.

To differentiate between purely visually evoked activity and brain response modulations due to the information displayed by the stimuli, we first showed grey arrows corresponding to all possible directions of turning (i.e. neutral warning stimulus). Then, one second later, we highlighted in green the arrow that corresponds to the car's expected direction (i.e. directional stimulus). This stimulus was always showed before the car reached the actual intersection.

Both the neutral and directional stimuli elicited clear visual evoked potentials. As expected, the responses after neutral stimuli (i.e. grey arrows) do not differ between correct and erroneous feedback. In contrast, we see clear differences in the EEG response over fronto-central areas when the directional stimulus does not match the user's intention; c.f., Figures $5(\mathrm{c})$ and $5(\mathrm{~d})$ for the data in the simulated and the real car, respectively. Statistical differences between error and correct conditions were observed between $200 \mathrm{~ms}$ and $600 \mathrm{~ms}$ after feedback.

To assess single-trial decoding, discriminant features (i.e. electrodes and time samples in the window [0.2 0.7] $\mathrm{s}$ after the directional cue) were selected using canonical variate analysis and fed to a LDA classifier (erroneous vs. correct feedback) [41]. In both simulated and real driving, the mean accuracy of the error-related decoding was about 0.68 across subjects (10-fold cross-validation).

Although it can be argued that this performance is not very high, it indicates the possibly of extracting meaningful information about the driver's assessment of the provided assistance. Interestingly, novel methods based on reinforcement learning have shown the possibility of improving human machine interaction even in the case of low detection rates of error-related potentials [40], [45]. 


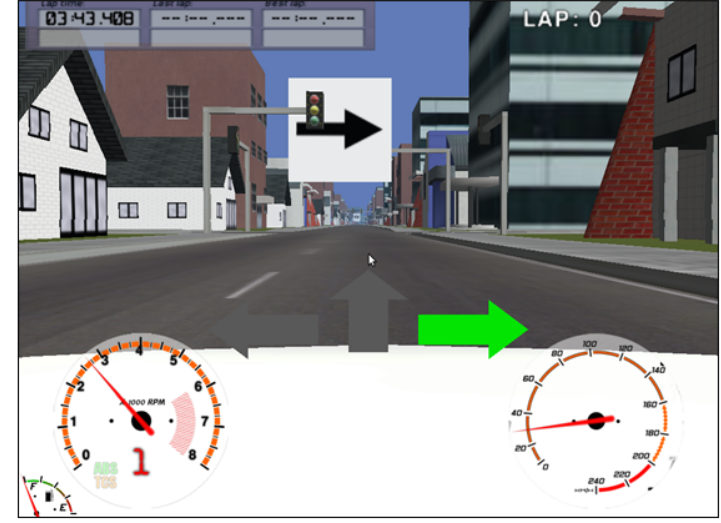

(a)

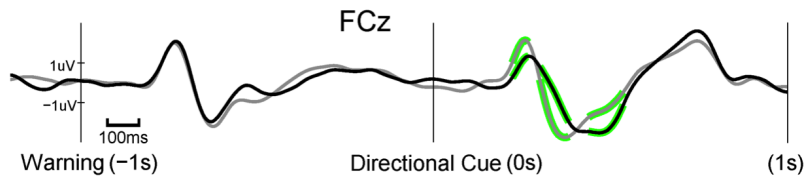

(c)

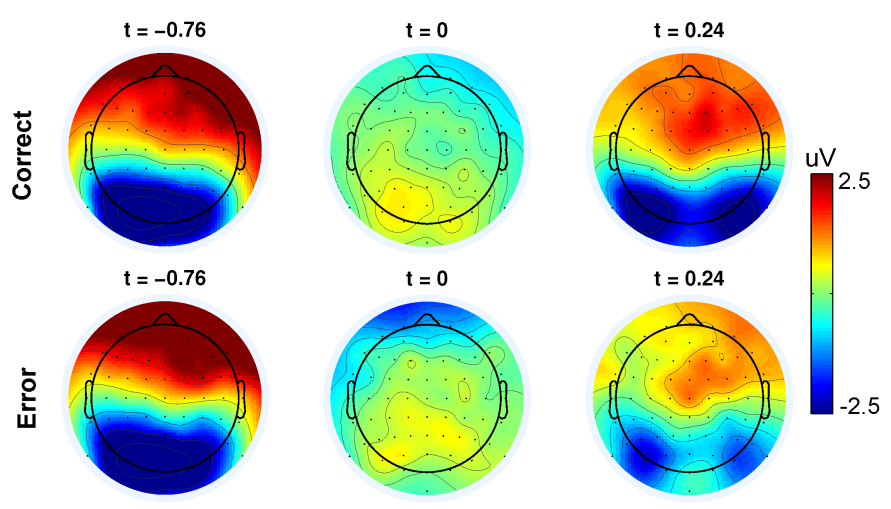

(b)

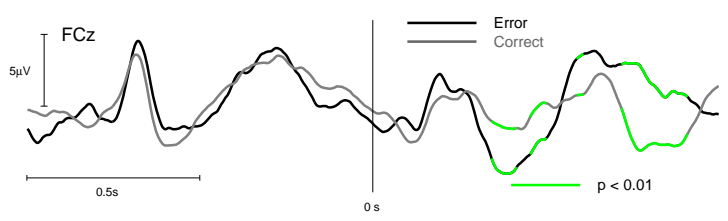

(d)

Fig. 5. Error-related potentials in driving scenarios [41]. (a) Experimental protocol. Screenshot of the driver's view when the directional cue is presented. (b) Grand average ERPs in the driving simulator for both the neutral and directional stimuli. Each plot depicts topographical activity at the onset of the directional cue $(\mathrm{t}=0)$ and the peak latencies after the warning and directional cues. (c) Grand average error-related potential (FCz electrode) in the driving simulator. (d) Error-related potential for one exemplar subject in the real car experiment.

\section{DISCUSSION}

Advances in sensing and control techniques have made it possible to endow cars with a growing set of features that assist drivers and can contribute to improve the driving experience. Some of these features can take control of specific aspects of the driving task (e.g cruise control, autonomous driving) or provide information to the driver to support specific maneuvers (e.g. lane departure). We propose that knowledge about the driver's cognitive states - decoded through a BMI - can contribute to better integrate these features to the driver's commands and preferences. Following the shared control approach, information about user's intention provided by the BMI and other sensors [12], [46]-[48] together with external context information (inferred from in-car sensors) [49]-[52] are taken into account to provide suitable driving assistance. In consequence, the intelligent car will provide timely and tailored assistance only when required; thus keeping the user in the loop.

As a first step towards this goal, we report that neural signatures of anticipation, movement preparation and error processing can be consistently found while subjects drive both in a simulator and a real car. Despite the increased level of noise in the signal that can be expected in this realistic scenario, obtained EEG correlates are well in line with those previously reported in simpler experimental paradigms. Deployment of BMI technologies seems thus promising. The reported EEGdecoding performances are sufficient as a proof of concept, yet, they are indicative of the necessity for improvements before translating them into automotive applications. We believe that to this end, the machine learning/computational intelligence community can significantly contribute. As previously discussed, the state-of-the-art BMI approaches are mainly focused on simple methods, what is perfectly justified in the classical paradigms created to isolate the cognitive processes of interest and minimize the sources of EEG artifacts. Moving to scenarios involving a complex and dynamic environment, and consequently more complex human behavior, motivates the need for methods suitable to tackle these new challenges -e.g. models robust to temporal variability of neural correlates, increased noise, interaction of neural processes. Some promising approaches include the use of novel features based on the connectivity across brain areas [6], [7], [15], [53]-[55] or the covariance across channels [56], deep learning [10], [57], as well as techniques for robust decoder training using limited samples such as transfer learning or semi-supervised approaches [9], [40], [58]-[61]. A recent review on current trends for EEG decoding in BMI applications can be found in reference [62].

Evidently, further studies are required to fully characterize neural activity related to driving tasks. In particular, it is of great importance to assess the actual decoding performance that can be expected from in-car BMIs in different conditions. For instance, comparing results in situations with different levels of traffic such as highways, secondary roads, or urban streets. In addition, other brain signals can potentially be exploited for similar purposes as those described in this paper. Recent works have focused on the decoding of brain correlates of visual attention processes [63]. Preliminary evidence suggests that these correlates can also be observed during driving [64], but further studies are required to validate these findings. At a certain point, the experimental paradigms would need to address the interaction of all these individual neural processes moving closer to the real-world driving 
scenario, where drivers are faced with dynamical and cluttered environment. In such scenario, driver's actions are somewhere in-between the cued and the self-paced actions.

Accordingly, further work is also needed on the assessment of the different processing techniques that can be applied to improve the reliability of these interfaces. Among these techniques, we can mention artifact detection and removal, as well as sensing technologies. The former are clearly needed as the driving task requires continuous eye and limb movements that can contaminate the recordings. It is worth noticing that in the results reported here we only applied simple techniques for artifact removal as threshold-based trial or channel rejection, so more advanced techniques are expected to improve the decoding performance [65]-[67]. Regarding the sensing technology, the usage of wearable, dry-electrode devices can have a considerable impact on the feasibility of using a BMI while driving. Significant improvements have been recently reported along these lines [54], [68]-[72], but further efforts have to be devoted to evaluate new sensing platforms in this application.

To summarize, in this work we introduced the possibility to decode cognition-related EEG activity of drivers complementing previous approaches for decoding information about the driver's state from both neural, physiological and behavioral. We envision hybrid systems where all these information sources are fused to reliably infer the driver's state. In consequence, another outstanding challenge lies on the characterization of the information that can be obtained from different sources such as EEG, EMG, eye/face tracking, driving profile, among others; and in consequence, the development of methods that fuse such information according to the specific context. Some efforts have been done in this line in other domains such as rehabilitation robotics [73] and activity recognition [74], and we foresee similar endeavours to be of great utility for automotive applications.

Finally, this information can be further leveraged by exploiting the sensing capabilities of intelligent cars. Adequate design of the human-car interaction can not only dynamically adjust the level of assistance provided to user, but also can provide feedback to better inform the user about the car's decisions or operation. In addition, this feedback may help to draw the user's attention to relevant information. This is not only beneficial for the driver to have better awareness of key information, but also can result in stronger neural and physiological responses (e.g. error-related potentials) that are in turn exploited by the BMI.

\section{ACKNOWLEDGMENT}

This work has been supported by Nissan Motor Co. Ltd., under the "Research on Brain Machine Interface for Drivers" project and the SNSF-funded NCCR Robotics. In-car experiments were possible thanks to the support of the Cantonal road service of Vaud, Switzerland. This paper only reflects the authors' view and funding agencies are not liable for any use that may be made of the information contained herein.

\section{REFERENCES}

[1] Scott Makeig, Klaus Gramann, Tzyy-Ping Jung, Terrence J Sejnowski, and Howard Poizner. Linking brain, mind and behavior. Int J Psychophysiol, 73(2):95-100, Aug 2009.

[2] Pedro M R Reis, Felix Hebenstreit, Florian Gabsteiger, Vinzenz von Tscharner, and Matthias Lochmann. Methodological aspects of EEG and body dynamics measurements during motion. Front Hum Neurosci, $8: 156,2014$

[3] Chin-Teng Lin, Shi-An Chen, Tien-Ting Chiu, Hong-Zhang Lin, and Li-Wei Ko. Spatial and temporal EEG dynamics of dual-task driving performance. J Neuroeng Rehabil, 8:11, 2011.

[4] Rami N. Khushaba, Sarath Kodagoda, Sara Lal, and Gamini Dissanayake. Driver drowsiness classification using fuzzy wavelet-packetbased feature-extraction algorithm. IEEE Trans Biomed Eng, 58(1):121131, Jan 2011.

[5] Chun-Hsiang Chuang, Li-Wei Ko, Tzyy-Ping Jung, and Chin-Teng Lin. Kinesthesia in a sustained-attention driving task. Neuroimage, 91:187202, May 2014.

[6] Chih-Sheng Huang, Nikhil R. Pal, Chun-Hsiang Chuang, and Chin-Teng Lin. Identifying changes in EEG information transfer during drowsy driving by transfer entropy. Frontiers in Human Neuroscience, 9:570, 2015.

[7] Wanzeng Kong, Weicheng Lin, Fabio Babiloni, Sanqing $\mathrm{Hu}$, and $\mathrm{Gi}-$ anluca Borghini. Investigating driver fatigue versus alertness using the granger causality network. Sensors, 15(8):19181-19198, 2015.

[8] Jon Touryan, Gregory Apker, Brent J. Lance, Scott E. Kerick, Anthony J. Ries, and Kaleb McDowell. Estimating endogenous changes in task performance from EEG. Frontiers in Neuroscience, 8:155, 2014.

[9] D. Wu, V. J. Lawhern, S. Gordon, B. J. Lance, and C. T. Lin. Driver drowsiness estimation from EEG signals using online weighted adaptation regularization for regression (OwARR). IEEE Transactions on Fuzzy Systems, PP(99):1-1, 2017.

[10] Rifai Chai, Sai Ho Ling, Phyo Phyo San, Ganesh R. Naik, Tuan N. Nguyen, Yvonne Tran, Ashley Craig, and Hung T. Nguyen. Improving EEG-based driver fatigue classification using sparse-deep belief networks. Frontiers in Neuroscience, 11:103, 2017.

[11] Gianluca Borghini, Laura Astolfi, Giovanni Vecchiato, Donatella Mattia, and Fabio Babiloni. Measuring neurophysiological signals in aircraft pilots and car drivers for the assessment of mental workload, fatigue and drowsiness. Neurosci Biobehav Rev, 44:58-75, Oct 2014.

[12] N. Li, J. J. Jain, and C. Busso. Modeling of driver behavior in real world scenarios using multiple noninvasive sensors. IEEE Transactions on Multimedia, 15(5):1213-1225, Aug 2013.

[13] S. Hart and L. Staveland. Human Mental Workload, chapter Development of NASA-TLX (Task Load Index): Results of Empirical and Theoretical Research, pages 139-83. North-Holland, 1988.

[14] Laura M Naismith, Jeffrey JH Cheung, Charlotte Ringsted, and Rodrigo B Cavalcanti. Limitations of subjective cognitive load measures in simulation-based procedural training. Medical education, 49(8):805814,2015

[15] G. N. Dimitrakopoulos, I. Kakkos, Z. Dai, H. Wang, K. Sgarbas, N. Thakor, A. Bezerianos, and Y. Sun. Functional connectivity analysis of mental fatigue reveals different network topological alterations between driving and vigilance tasks. IEEE Transactions on Neural Systems and Rehabilitation Engineering, 26(4):740-749, April 2018.

[16] Sheng-Hsiou Hsu and Tzyy-Ping Jung. Monitoring alert and drowsy states by modeling EEG source nonstationarity. Journal of neural engineering, 14(5):056012, 2017.

[17] Thien Nguyen, Sangtae Ahn, Hyojung Jang, Sung Chan Jun, and Jae Gwan Kim. Utilization of a combined EEG/NIRS system to predict driver drowsiness. Scientiific Reports, 7:43933, 2017.

[18] Mervyn V.M. Yeo, Xiaoping Li, Kaiquan Shen, and Einar Wilder-Smith. Can SVM be used for automatic EEG detection of drowsiness during car driving? In Safety Science, volume 47, pages 115-124, 012009.

[19] Ning-Han Liu, Cheng-Yu Chiang, and Hsiang-Ming Hsu. Improving driver alertness through music selection using a mobile EEG to detect brainwaves. Sensors, 13(7):8199-8221, 2013.

[20] Chin-Teng Lin, Li-Wei Ko, and Tzu-Kuei Sheng. Computational intelligent brain computer interaction and its applications on driving cognition. IEEE Computational Intelligence Magazine, 4(4):32-46, 2009.

[21] Y. T. Liu, Y. Y. Lin, S. L. Wu, C. H. Chuang, and C. T. Lin. Brain dynamics in predicting driving fatigue using a recurrent self-evolving fuzzy neural network. IEEE Transactions on Neural Networks and Learning Systems, 27(2):347-360, Feb 2016. 
[22] Tao Liu, Matthew Pelowski, Changle Pang, Yuanji Zhou, and Jianfeng Cai. Near-infrared spectroscopy as a tool for driving research. Ergonomics, 59(3):368-379, 2016.

[23] Javier Izquierdo-Reyes, Ricardo A. Ramirez-Mendoza, and Martin R. Bustamante-Bello. A study of the effects of advanced driver assistance systems alerts on driver performance. International Journal on Interactive Design and Manufacturing (IJIDeM), 12(1):263-272, Feb 2018.

[24] Stefan Haufe, Matthias S Treder, Manfred F Gugler, Max Sagebaum, Gabriel Curio, and Benjamin Blankertz. EEG potentials predict upcoming emergency brakings during simulated driving. J Neural Eng, 8(5):056001, Jul 2011.

[25] Stefan Haufe, Jeong-Woo Kim, Il-Hwa Kim, Andreas Sonnleitner, Michael Schrauf, Gabriel Curio, and Benjamin Blankertz. Electrophysiology-based detection of emergency braking intention in real-world driving. J Neural Eng, 11(5):056011, Aug 2014.

[26] Il-Hwa Kim, Jeong-Woo Kim, Stefan Haufe, and Seong-Whan Lee. Detection of braking intention in diverse situations during simulated driving based on EEG feature combination. J Neural Eng, 12(1):016001, Feb 2015.

[27] F. Lotte, M. Congedo, A. Lécuyer, F. Lamarche, and B. Arnaldi. A review of classification algorithms for EEG-based brain-computer interfaces. J Neural Eng, 4(2):R1-R13, Jun 2007.

[28] Benjamin Blankertz, Steven Lemm, Matthias Treder, Stefan Haufe, and Klaus robert Mller. Single-trial analysis and classification of erp components - a tutorial, 2010.

[29] W. G. Walter, R. Cooper, V. J. Aldridge, W. C. McCallum, and A. L. Winter. Contingent negative variation: An electric sign of sensorimotor association and expectancy in the human brain. Nature, 203:380-4, Jul 251964.

[30] Gangadhar Garipelli, Ricardo Chavarriaga, and José del R. Millán. Single trial analysis of slow cortical potentials: a study on anticipation related potentials. J Neural Eng, 10(3):036014, Apr 2013.

[31] Zahra Khaliliardali, Ricardo Chavarriaga, Lucian Andrei Gheorghe, and José del R. Millán. Action prediction based on anticipatory brain potentials during simulated driving. J Neural Eng., 12(6):066006, 2015.

[32] Xavier Perrin, Ricardo Chavarriaga, Francis Colas, Roland Siegwart, and José del R. Millán. Brain-coupled interaction for semi-autonomous navigation of an assistive robot. Robotics and Autonomous Systems, 58(12):1246-1255, 2010

[33] B. Libet, C. A. Gleason, E. W. Wright, and D. K. Pearl. Time of conscious intention to act in relation to onset of cerebral activity (readiness-potential). the unconscious initiation of a freely voluntary act. Brain, 106 (Pt 3):623-642, Sep 1983.

[34] Imran Khan Niazi, Ning Jiang, Olivier Tiberghien, Jrgen Feldbk Nielsen, Kim Dremstrup, and Dario Farina. Detection of movement intention from single-trial movement-related cortical potentials. J Neural Eng, 8(6):066009, Dec 2011

[35] Eileen Lew, Ricardo Chavarriaga, Stefano Silvoni, and José del R. Millán. Detection of self-paced reaching movement intention from EEG signal. Frontiers in Neuroengineering, 5(13), 2012.

[36] Tom Carlson, Robert Leeb, Ricardo Chavarriaga, and José del R. Millán. Online modulation of the level of assistance in shared control systems. In Proceedings of the IEEE International Conference on Systems Man and Cybernetics (SMC 2012), pages 3321-3326, 2012.

[37] Ricardo Chavarriaga, Xavier Perrin, Roland Siegwart, and José del R. Millán. Anticipation- and error-related EEG signals during realistic human-machine interaction: A study on visual and tactile feedback. In 34th International Conference of the IEEE Engineering in Medicine and Biology Society (EMBC'12), pages 6723-6726, 2012.

[38] Tom Carlson and José del R. Millán. Brain-controlled wheelchairs: A robotic architecture. IEEE Robotics and Automation Magazine, 20(1):65-73, 2013.

[39] Ricardo Chavarriaga, Aleksander Sobolewski, and José del R. Millán. Errare machinale est: the use of error-related potentials in brain-machine interfaces. Front Neurosci, 8:208, 2014.

[40] Iñaki Iturrate, Ricardo Chavarriaga, Luis Montesano, Javier Minguez, and José del R. Millán. Teaching brain-machine interfaces as an alternative paradigm to neuroprosthetics control. Scientific Reports, 5:13893, 2015.

[41] H. Zhang, R. Chavarriaga, Z. Khaliliardali, L. Gheorghe, I. Iturrate, and José del R. Millán. EEG-based decoding of error-related brain activity in a real-world driving task. J Neural Eng, 12(6):066028, Nov 2015.

[42] Zahra Khaliliardali, Ricardo Chavarriaga, Zhang Huaijian, Lucian Andrei Gheorghe, and José del R. Millán. Single trial classification of neural correlates of anticipatory behavior during real car driving. 6th International Brain-Computer Interface Meeting, Asilomar, California, USA, 2016.
[43] Lucian Andrei Gheorghe, Ricardo Chavarriaga, and José del R. Millán. Steering Timing Prediction in a Driving Simulator Task. In Proceedings of the 35th Annual International Conference of the IEEE Engineering in Medicine and Biology Society, pages 6913-6916, 2013.

[44] Huaijian Zhang, Ricardo Chavarriaga, Lucian Andrei Gheorghe, and José del R. Millán. Brain correlates of lane changing reaction time in simulated driving. In IEEE International Conference on Systems, Man, and Cybernetics, 2015.

[45] Victor Shih, David C. Jangraw, Paul Sajda, and Sameer Saproo. Towards personalized human $\mathrm{AI}$ interaction - adapting the behavior of AI agents using neural signatures of subjective interest. arXiv:1709.04574, 2017.

[46] E. Murphy-Chutorian and M. M. Trivedi. Head pose estimation and augmented reality tracking: An integrated system and evaluation for monitoring driver awareness. IEEE Transactions on Intelligent Transportation Systems, 11(2):300-311, June 2010.

[47] Juan Carmona, Fernando García, David Martín, Arturo de la Escalera, and José María Armingol. Data fusion for driver behaviour analysis. Sensors, 15(10):25968-25991, 2015.

[48] Enrique David Martí, David Mart' in, Jesús García, Arturo de la Escalera, José Manuel Molina, and José María Armingol. Context-aided sensor fusion for enhanced urban navigation. Sensors, 12(12):1680216837, 2012.

[49] Moises Diaz, Pietro Cerri, Giuseppe Pirlo, Miguel A. Ferrer, and Donato Impedovo. A Survey on Traffic Light Detection, pages 201-208. Springer International Publishing, Cham, 2015.

[50] Guohua Wang and Qiong Liu. Far-infrared based pedestrian detection for driver-assistance systems based on candidate filters, gradient-based feature and multi-frame approval matching. Sensors, 15(12):32188$32212,2015$.

[51] Juan Jesús Castillo Aguilar, Juan Antonio Cabrera Carrillo, Antonio Jesús Guerra Fernández, and Enrique Carabias Acosta. Robust road condition detection system using in-vehicle standard sensors. Sensors, 15(12):32056-32078, 2015.

[52] Chun-Fu Lin, Chin-Sheng Chen, Wen-Jyi Hwang, Chih-Yen Chen, ChiHung Hwang, and Chun-Li Chang. Novel outline features for pedestrian detection system with thermal images. Pattern Recognition, 48(11):3440 $-3450,2015$

[53] Huaijian Zhang, Ricardo Chavarriaga, and José del R. Millán. Discriminant brain connectivity patterns of performance monitoring at average and single-trial levels. Neuroimage, 120:64-74, Jul 2015.

[54] Tim R. Mullen, Christian A E. Kothe, Yu Mike Chi, Alejandro Ojeda, Trevor Kerth, Scott Makeig, Tzyy-Ping Jung, and Gert Cauwenberghs. Real-time neuroimaging and cognitive monitoring using wearable dry EEG. IEEE Trans Biomed Eng, 62(11):2553-2567, Nov 2015.

[55] Martin Billinger, Clemens Brunner, and Gernot R. Müller-Putz. Online visualization of brain connectivity. J Neurosci Methods, 256:106-116, Sep 2015.

[56] Marco Congedo, Alexandre Barachant, and Rajendra Bhatia. Riemannian geometry for EEG-based brain-computer interfaces; a primer and a review. Brain-Computer Interfaces, 4(3):155-174, 2017.

[57] Robin Tibor Schirrmeister, Jost Tobias Springenberg, Lukas Dominique Josef Fiederer, Martin Glasstetter, Katharina Eggensperger, Michael Tangermann, Frank Hutter, Wolfram Burgard, and Tonio Ball. Deep learning with convolutional neural networks for EEG decoding and visualization. Human Brain Mapping, 38(11):5391-5420, 2017.

[58] Pieter-Jan Kindermans, Martijn Schreuder, Benjamin Schrauwen, KlausRobert Müller, and Michael Tangermann. True zero-training braincomputer interfacing: an online study. PLOS ONE, 9(7):1-13, 072014.

[59] R. G. F. Soares, H. Chen, and X. Yao. A cluster-based semisupervised ensemble for multiclass classification. IEEE Transactions on Emerging Topics in Computational Intelligence, 1(6):408-420, Dec 2017.

[60] A. Saha, A. Konar, and A. K. Nagar. EEG Analysis for Cognitive Failure Detection in Driving Using Type-2 Fuzzy Classifiers. IEEE Transactions on Emerging Topics in Computational Intelligence, 1(6):437-453, December 2017.

[61] A. Gupta, Y. S. Ong, and L. Feng. Insights on transfer optimization: Because experience is the best teacher. IEEE Transactions on Emerging Topics in Computational Intelligence, 2(1):51-64, Feb 2018.

[62] Fabien Lotte, Laurent Bougrain, Andrzej Cichocki, Maureen Clerc, Marco Congedo, Alain Rakotomamonjy, and Florian Yger. A review of classification algorithms for EEG-based brain-computer interfaces: A 10-year update. Journal of Neural Engineering, 2018.

[63] Luca Tonin, Robert Leeb, Aleksander Sobolewski, and José del R. Millán. An online EEG BCI based on covert visuospatial attention in absence of exogenous stimulation. J Neural Eng, 10(5):056007, Oct 2013. 
[64] Hadrian Renold, Ricardo Chavarriaga, Lucian Andrei Gheorghe, and José del R. Millán. EEG correlates of active visual search during simulated driving: An exploratory study. In IEEE International Conference on Systems, Man, and Cybernetics, San Diego, USA, 2014

[65] A. Schlögl, C. Keinrath, D. Zimmermann, R. Scherer, R. Leeb, and G. Pfurtscheller. A fully automated correction method of EOG artifacts in EEG recordings. Clin Neurophysiol, 118(1):98-104, Jan 2007.

[66] Joseph T Gwin, Klaus Gramann, Scott Makeig, and Daniel P Ferris. Removal of movement artifact from high-density EEG recorded during walking and running. J Neurophysiol, 103(6):3526-3534, Jun 2010.

[67] S. K. Goh, H. A. Abbass, K. C. Tan, A. Al-Mamun, C. Wang, and C. Guan. Automatic EEG Artifact Removal Techniques by Detecting Influential Independent Components. IEEE Transactions on Emerging Topics in Computational Intelligence, 1(4):270-279, August 2017.

[68] M. Lopez-Gordo, D. Morillo, and F. Valle. Dry EEG electrodes. Sensors, 14(7):1284712870, Jul 2014.

[69] W. David Hairston, Keith W. Whitaker, Anthony J. Ries, Jean M. Vettel, J. Cortney Bradford, Scott E. Kerick, and Kaleb McDowell. Usability of four commercially-oriented EEG systems. J Neural Eng, 11(4):046018, Aug 2014.

[70] John K. Zao, Tchin-Tze Gan, Chun-Kai You, Cheng-En Chung, Yu-Te Wang, Sergio José Rodríguez Méndez, Tim Mullen, Chieh Yu, Christian Kothe, Ching-Teng Hsiao, San-Liang Chu, Ce-Kuen Shieh, and TzyyPing Jung. Pervasive brain monitoring and data sharing based on multitier distributed computing and linked data technology. Front Hum Neurosci, 8:370, 2014.

[71] Wei-Long Zheng and Bao-Liang Lu. A multimodal approach to estimating vigilance using EEG and forehead EOG. Journal of neural engineering, 14(2):026017, 2017.

[72] O. Dehzangi and C. Williams. Towards multi-modal wearable driver monitoring: Impact of road condition on driver distraction. In 2015 IEEE 12th International Conference on Wearable and Implantable Body Sensor Networks (BSN), pages 1-6, June 2015.

[73] Domen Novak, Ximena Omlin, Rebecca Leins, and Robert Riener. Predicting targets of human reaching motions using different sensing technologies. IEEE Trans Biomed Eng, 60:2645-2654, May 2013.

[74] Daniel Roggen, Paul Lukowicz, Alois Ferscha, José del R. Millán, Gerhard Tröster, and Ricardo Chavarriaga. Opportunistic human activity and context recognition. Computer, 46(2):36-45, 2013. 